\title{
Determinantes da distribuição geográfica de Callithrix flaviceps (Thomas) (Primates, Callitrichidae)
}

\author{
Carlos E. V. Grelle ${ }^{1,2}$ \& Rui Cerqueira ${ }^{1}$
}

${ }^{1}$ Laboratório de Vertebrados, Departamento de Ecologia, Universidade Federal do Rio de Janeiro. 21941-590 Rio de Janeiro, Rio de Janeiro, Brasil.

2 Autor correspondente. E-mail: grellece@biologia.ufri.br

\begin{abstract}
Determinants of geographical range of Callithrix flaviceps (Thomas) (Primates, Callitrichidae). The climate and the vegetation are known determinant of the geographical range of several species of mammals. Here it was analized the role of the climate and the vegetation as limits of geographical range of the marmosets Callithrix flaviceps (Thomas, 1903). For each locality of occurrence, averages estimates of nine climatic variables and ecoregion data were recorded. A non-linear method (logistic regression) was used to model the climatic geographical distribution. Vegetation analysis was done with data from the digital map of ecoregions. The overlap of the climatic distribution map with ecoregion map suggested that both factors have importance in the limits of the geographical range of $C$. flaviceps. The role of interspecific interactions as limits to geographical range of $C$. flaviceps is also discussed.
\end{abstract}

KEY WORDS. Atlantic forest; biogeography; climate; ecoregion; logistic regression.

RESUMO. O clima e a vegetação são conhecidos determinantes das distribuições geográficas de várias espécies de mamíferos. Neste estudo foi testada a importância do clima e da vegetação como fatores limitantes da distribuição geográfica do sagui Callithrix flaviceps (Thomas, 1903). As análises com o clima foram feitas a partir de nove fatores climáticos, estimados para cada localidade de ocorrência da espécie em estudo. Métodos não lineares (regressão logística) foram usados para modelar a distribuição geográfica a partir dos dados climáticos. As análises de vegetação foram feitas a partir do mapa digital das eco-regiões. A sobreposição dos modelos de distribuição climática com os mapas dos tipos de eco-regiões sugeriram que estes dois fatores são limitantes na distribuição geográfica de Callithrix flaviceps. Foi ainda discutida a importância das interações interespecíficas como limitantes da distribuição geográfica de Callithrix flaviceps.

PALAVRAS-CHAVE. Biogeografia; clima; eco-região; Mata Atlântica; regressão logística.

Os limites de distribuição geográfica das espécies, sejam eles bióticos ou abióticos, são, muitas vezes, difíceis de serem identificados. As dificuldades vão desde a falta de amostras suficientes para se detectar as extensões e descontinuidades, até a dinâmica espaço-temporal que as espécies apresentam nas suas distribuições geográficas (TAYLOR \& TAYLOR 1979, CERQUeIra 1985, 1995). As espécies, contudo, não se distribuem aleatoriamente, indicando que alguns, ou muitos, fatores ambientais podem determinar os limites das distribuições (Endler 1982, Rapoport 1982, Myers \& Giller 1988a, b, Cerqueira 1995). A busca destes fatores é, provavelmente, mais importante do que o próprio limite das distribuições, já que eles permitem a construção de modelos preditivos, permitindo que se teste empiricamente a relevância deles para determinar as distribuições. Além disso, os modelos construídos a partir de fatores ambientais possibilitam o estudo das mudanças nas distribuições geográficas em cenários pretéritos e futuros.
O clima e a vegetação são determinantes conhecidos das distribuições geográficas e das densidades de várias espécies de mamíferos (e.g. Cerqueira 1985, Walker 1990, LindnMayer et al. 1991, Jefrree \& Jefrree 1994, LaW 1994, Cerqueira 1995, Cerqueira et al. 1998, JaCKSon \& Claridge 1999, Grelle 2003), aves (e.g. Cueto \& Casenave 1999) e répteis (por exemplo Vanzolin 1970, Nix 1986, McKenney et al. 1998). Os pontos empíricos de ocorrência das espécies podem ser analisados a partir de seus fatores climáticos, de vegetação e de altitude (CERQUEIRA, 1985, 1995, Nix 1986, ScotT et al. 2002). Esta metodologia considera que comparações entre os fatores ambientais de espécies filogeneticamente próximas poderiam indicar quais deles estariam influenciando os limites das distribuições geográficas dos táxons. Tal procedimento permite uma estimativa das distribuições geográficas potenciais, possibilitando a determinação dos limites e descontinuidades das delas (Cerqueira, 1985, 1995, Cerqueira et al. 1998). Seguindo 
este raciocínio, cada espécie tem um perfil climático que pode ser determinado, não existindo duas espécies com exatamente os mesmos valores (média, desvio padrão, amplitudes máxima e mínima) para os fatores climáticos. A idéia central, de o que o clima pode ser usado para predizer os limites de distribuição de uma espécie, está baseada no conceito dos homoclimas. Segundo este conceito, o clima pode ser mapeado porque existem localidades com condições climáticas similares, permitindo que se tracem isolinhas.

Existem quatro espécies de sagüis endêmicos na Mata Atlântica: Callithrix aurita (E. geoffroy, 1812), C. flaviceps (Thomas, 1903), C. geoffroyi (Humboldt, 1812) e C. kuhlii (Wied, 1826). Os dois primeiros são considerados como espécies que ocorrem principalmente nas montanhas do sudeste brasileiro (Ferrari et al. 1996, Rylands et al. 1996, Cerqueira et al. 1998), sendo incluídos entre as espécies ameaçadas de extinção, devido principalmente à destruição da Mata Atlântica (FONSECA et al. 1994, Machado et al. 1998, Bergallo et al. 2000). Poucos estudos de campo foram feitos sobre estas duas espécies, mas sabese que, entre os calitriquídeos que ocorrem na Mata Atlântica, elas são as espécies que habitam as áreas com as condições climáticas mais extremas (Ferrari et al. 1996, CorrêA et al. 2000). Sendo assim, é possível que o clima seja um fator limitante na distribuição geográfica desses dois callithiquídeos que ocorrem nas montanhas do sudeste brasileiro.

Os limites das distribuições geográficas de Callithrix Erxleben, 1777 são pouco conhecidos. As hipóteses vigentes argumentam que os limites geográficos seriam, basicamente, acidentes geográficos, como rios e montanhas (HershKovitz 1977, KinSEy 1982, Rylands et al. 1996). CERQUeira et al. (1998) avaliaram as distribuições potenciais, a partir de dados climáticos e de vegetação, para C. aurita no Estado do Rio de Janeiro.

Estudos taxonômicos prévios sobre as espécies de Callithrix do grupo jacchus sugeriram que todas as formas seriam subespécies de C. jacchus (Hershrovitz 1977). Posteriormente, Vivo (1991) fez uma revisão de todo gênero Callithrix e concluiu que o grupo jacchus seria formado por cinco espécies: $C$. aurita, C. flaviceps, C. geoffroyi, C. penicillata (É. Geoffroy, 1812) e C. jacchus (Linnaeus, 1758) -, não reconhecendo C. kuhlii como táxon válido. Contudo, estudos mais recentes demonstraram que C. kuhlii é uma espécie válida (NATori 1990, 1994). As quatro espécies de Callithrix endêmicos da Mata Atlântica (C. aurita, C. flaviceps, C. geoffroyi e C. kuhlii) são parapátricas e com pequenas zonas de hibridação entre algumas das formas (Vivo 1991, RYLANDS et al. 1996).

Várias técnicas de estatística multivariada têm sido usadas para se estudar a relação entre os fatores ambientais e as distribuições das espécies (e.g. Cerqueira 1985, 1995, Hill 1991, Cerqueira et al. 1998, Manel et al. 1999 Andrew \& O'Brien 2000). Dentro deste contexto, o uso de modelos de regressão, particularmente os de regressão logística, são adequados porque este método permite que se analise o efeito de uma ou mais variáveis independentes (discretas ou contínuas) sobre uma variá- vel dependente dicotômica, representando a presença (1) ou ausência (0) da espécie (Hosmer \& Lemeshow 1989, Manly et al. 1993). Além disso, os modelos de regressão logística permitem ainda que se tracem curvas de probabilidade da distribuição espacial das espécies (veja exemplos em WaLker 1990, Bustamante 1997, Rodriguez \& ANDrén 1999, SCOTT et al. 2002).

Os objetivos deste estudo foram: I) compilar as localidades de ocorrência das quatro espécies de Callithrix; II) descrever o perfil climático de C. flaviceps; III) fazer a distribuição potencial climática desta espécie a partir dos fatores climáticos, IV) mapear e sobrepor a distribuição potencial climática e da vegetação e; IV) discutir quais seriam os fatores que parecem determinar as extensões geográficas de $C$. flaviceps, e com isso testar a hipótese de que o clima é um fator limitante à distribuição geográfica desta espécie.

\section{MATERIAL E MÉTODOS}

\section{Bases de dados}

Os dados das localidades foram compilados nos espécimes depositados em museus de história natural e na literatura. As coordenadas geográficas das localidades foram obtidas em United States (1963), Painter \& Taylor (1991) e Vanzolini (1992). Estimativas médias de nove variáveis climáticas foram registradas para cada localidade, sendo elas: temperatura média (Tmed), temperatura média mínima (Tmín), temperatura média máxima (Tmáx), temperatura mínima absoluta (Minab), temperatura máxima absoluta (Maxab), precipitação total anual (Prec), nebulosidade anual (Neb), umidade relativa anual (Ur) e dias de chuva (Dc). Estas informações foram obtidas usando o sistema de dados climáticos do Laboratório de Vertebrados, Departamento de Ecologia, Universidade Federal do Rio de Janeiro. Este sistema foi montado a partir de vários dados climáticos, sendo que a maior parte deles vieram do "Atlas Climatológico do Brasil" (BRASIL 1969), nos permitindo obter os valores para as variáveis climáticas em uma escala fina (minuto a minuto) para qualquer localidade no Brasil.

Os dados de vegetação de cada localidade, por espécie, foram obtidos no mapa digital das eco-regiões propostas para a América Latina e Central (DinersteIn et al. 1995). As divisões e os limites das eco-regiões são semelhantes às formações vegetacionais do mapa de vegetação do IbGE (1993). De acordo com Dinerstein et al. (1995), eco-região seriam unidades biogeográficas, representando comunidades vegetais que compartilham a maioria das suas espécies, dinâmicas e processos ecológicos.

\section{Análises dos dados}

Os limites climáticos da distribuição geográfica de $C$. flaviceps foram investigados por regressão logística. Este método permite a comparação entre as localidades onde a espécie ocorre contra aquelas onde ela não ocorre. Desta forma, podese obter uma resposta das variáveis independentes em relação a presença (representada por 1) e a ausência (representada por 0) de cada espécie estudada. Sendo assim, os dados climáticos 
das localidades de C. flaviceps foram contrastados com os dados climáticos das localidades onde esta espécie não ocorre (localidades de C. aurita, C. geoffroyi e C. kuhli).

O modelo preditivo de regressão logística múltipla usado seguiu a equação: $\operatorname{Logit}(\mathrm{p})=\beta_{0}+\beta_{1} \mathrm{X}_{1}+\beta_{2} \mathrm{X}_{2}+\ldots+\beta_{\mathrm{p}} \mathrm{X}_{\mathrm{p}}$; onde: $\beta_{0}$ é uma constante e $\beta_{1} \ldots \beta_{\mathrm{p}}$ são coeficientes das variáveis independentes $X_{1} \ldots X_{\mathrm{p}}$ (Hosmer \& Lemeshow 1989). A partir da equação descrita acima, várias probabilidades podem ser estimadas por: $\mathrm{P}=\left(e^{\text {Logit(p) }}\right) /\left(1+e^{\text {Logit( } \mathrm{p})}\right)$; onde: $\mathrm{P}$ é a probabilidade de ocorrência da espécie (de 0 a 1 ) e $e$ é a base dos logaritmos naturais.

O procedimento foi o passo-a-passo, opção progressiva ("forward"), com critério 0,1 para inclusão e exclusão das variáveis. As estimativas dos parâmetros foram feitas por técnicas de máxima verossimilhança (Hosmer \& Lemeshow 1989). O procedimento passo-a-passo foi escolhido para minimizar os problemas de colinearidade, já que os dados climáticos são, por essência, correlacionados.

Foram feitas, então, curvas de probabilidade de ocorrência usando o programa Surfer 8.0 (Golden 2002) a partir das variáveis climáticas selecionadas na regressão logística. Os resultados das análises, com os limites de distribuição definidos pelas eco-regiões e pelo clima, foram sobrepostos usando o programa ArcMap 8.2 (EsRI 2002).

\section{RESULTADOS}

Ao todo foram compiladas 61 localidades de Callithrix aurita, 25 de C. flaviceps, 62 de C. geoffroyi, 23 de C. kuhlii, quatro de híbridos entre $C$. aurita e $C$. flaviceps, três de híbridos entre $C$. flaviceps e C. geoffroyi, três de híbridos entre C. geoffroyi e C. penicillata e um híbrido entre C. kuhlii com C. penicillata (Fig. 1). A lista completa com todas as localidades pode ser obtida com o primeiro autor. Duas das localidades dos híbridos entre C. flaviceps e C. geoffroyi são as mesmas das formas parentais de C. flaviceps e uma é a mesma localidade das formas parentais de C. geoffroyi. Para evitar repetição de localidades, as três foram excluídas das análises. Para evitar outra repetição de localidades, as quatro localidades dos híbridos entre $C$. aurita e C. flaviceps foram somadas somente aquelas das formas parentais de C. flaviceps. Então, as análises foram feitas com 29 localidades de C. flaviceps $(25+$ quatro dos híbridos), 61 de C. aurita, 65 de C. geoffroyi (62 + três híbridos) e de 24 para C. kuhlii ( 23 + uma de híbrido).

A tabela I apresenta os valores estimados das variáveis climáticas para C. flaviceps. O modelo obtido na regressão logística para C. flaviceps obteve uma concordância de 95,6\% entre as probabilidades previstas e as observadas. As variáveis selecionadas no modelo passo a passo foram: Temperatura mínima absoluta, Precipitação, Umidade Relativa e Dias de Chuva (Tab. II). A variável umidade relativa teve uma relação positiva com a presença de $C$. flaviceps, o que denota uma preferência desta espécie por localidades úmidas (Tab. II).

Callithrix flaviceps ocorre em três tipos de eco-regiões, sendo que mais de $50 \%$ das localidades estão situadas em florestas ombrófilas (Fig. 2).

Revista Brasileira de Zoologia 23 (2): 414-420, junho 2006

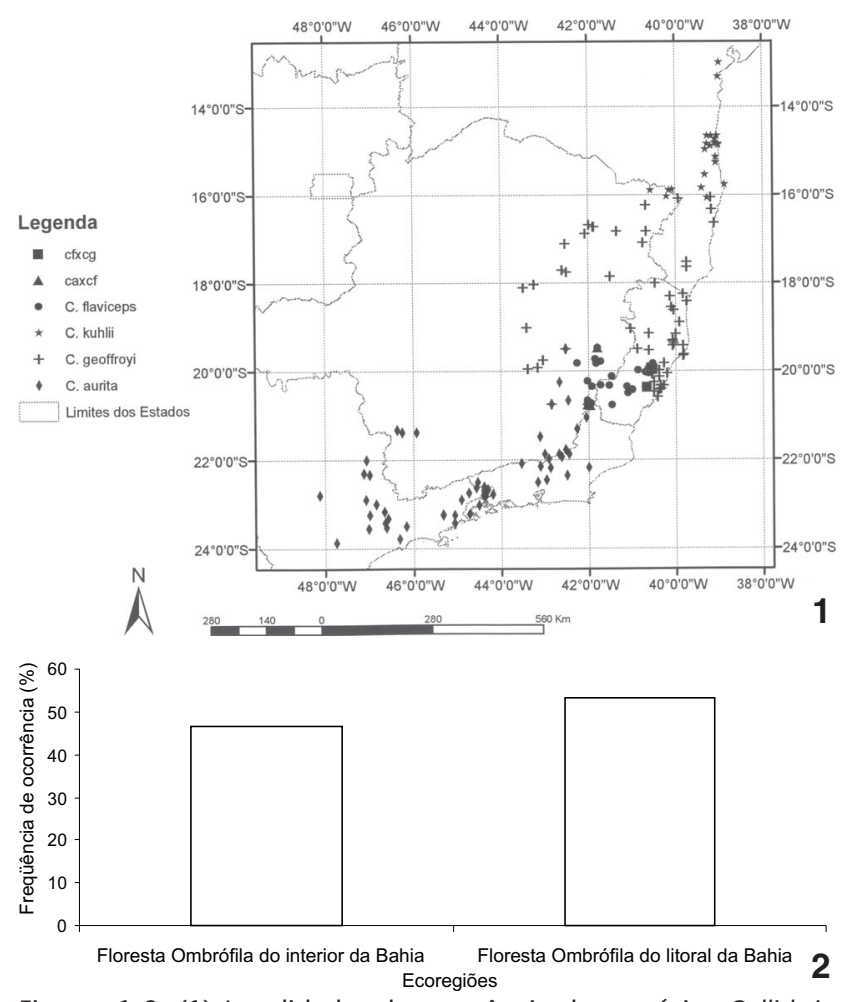

Figuras 1-2. (1) Localidades de ocorrência das espécies Callithrix aurita, C. flaviceps, C. geoffroyi, C. kuhlii, e dos híbridos entre C. flaviceps e C. geoffroyi e (cfxcg) e entre C. aurita e C. flaviceps (caxcf); (2) freqüência de ocorrência de $C$. flaviceps nas eco-regiões.

Tabela I. Estatísticas básicas das variáveis climáticas das localidades de ocorrência de Callithrix flaviceps. (DP) Desvio padrão, (Dc) dias de chuva, (Neb) nebulosidade anual, (N) número de localidades, (Prec) precipitação total anual, (Tmed) temperatura média, (Tmín) temperatura média mínima, (Tmáx) temperatura média máxima, (Tminab) temperatura mínima absoluta, (Tmaxab) temperatura máxima absoluta, (Ur) umidade relativa anual. Temperaturas fornecidas em graus Celsius $\left({ }^{\circ} \mathrm{C}\right)$.

\begin{tabular}{crrcrr}
\hline Variável & Média & DP & Mínimo & Máximo & $\mathrm{N}$ \\
\hline Tméd & 21,1 & 1,2 & 18,6 & 23,0 & 29 \\
Tmín & 16,5 & 1,7 & 14,0 & 19,3 & 29 \\
Tmáx & 27,6 & 0,9 & 25,1 & 28,8 & 29 \\
Minab & 2,2 & 2,6 & $-1,7$ & 6,2 & 29 \\
Maxab & 36,9 & 1,8 & 32,7 & 39,9 & 29 \\
Prec & 1226,9 & 176,6 & 999,9 & 1528,9 & 29 \\
Neb & 6,2 & 0,3 & 5,5 & 7,0 & 29 \\
Ur & 82,0 & 2,0 & 79,5 & 85,7 & 29 \\
Dc & 103,5 & 14,9 & 90,0 & 147,0 & 29 \\
\hline
\end{tabular}

A figura 3 apresenta as curvas de probabilidade da distribuição climática predita a partir das variáveis climáticas 


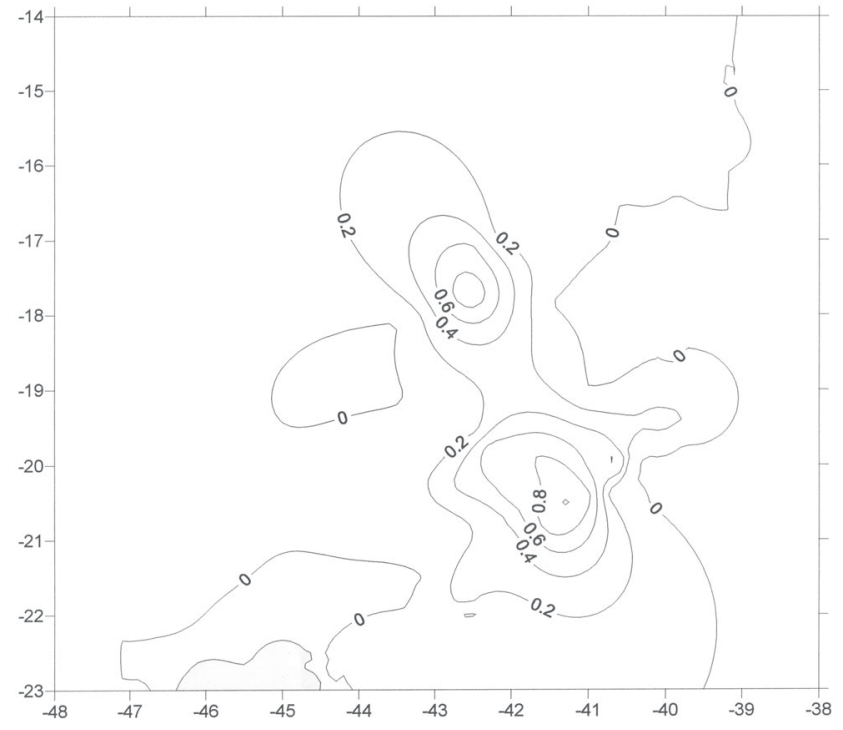

Figura 3. Distribuição predita, em probabilidades de 0 a 1 , para Callithrix flaviceps a partir das variáveis climáticas selecionadas no modelo de regressão logística (veja o texto). Os eixos estão representando os graus de latitude e longitude.

Tabela II. Variáveis climáticas significativas no modelo de regressão logística para a distribuição de Callithrix flaviceps.

\begin{tabular}{lcrrc}
\hline Variável & Parâmetro estimado & Erro Padrão & Wald $\chi^{2}$ & $P$ \\
\hline Constante & $-46,3109$ & 11,48350 & 16,2637 & 0,0001 \\
Minab & $-0,6417$ & 0,14630 & 19,2417 & 0,0001 \\
Prec & $-0,0087$ & 0,00229 & 14,4900 & 0,0001 \\
Ur & 0,8057 & 0,17290 & 21,7037 & 0,0001 \\
Dc & $-0,0680$ & 0,01770 & 14,6962 & 0,0001 \\
\hline
\end{tabular}

selecionadas nas regressões logísticas para C. flaviceps. Nas figuras 4 e 5 são sobrepostas as localidades de ocorrência, os tipos de eco-regiões e as curvas de probabilidade preditas para a distribuição climática.

\section{DISCUSSÃO}

A distribuição geográfica de $C$. flaviceps parece ser limitada pelos contornos da Mata Atlântica. De fato, todas as localidades estão situadas em eco-regiões que fazem parte da Mata Atlântica.

Uma das premissas da regressão logística é a de que não existem dúvidas quanto aos dados que são ausências (0) ou presenças (1) (Hosmer \& LeMeshow 1989). Este pode ser um problema nos estudos que usam regressão logística para investigar seleção de habitat, especialmente em escala de microhabitat, desde que a ausência (0) pode ser causada por falhas na amostragem ou ainda por baixas densidades (NorTh \& ReYNolds 1996). Neste sentido, o método de regressão logística parece ser especialmente adequado para modelar a distribuição de espécies parapátricas, desde que a sobreposição destas espécies seja quase que inexistente (ENDLER 1977). Neste caso, os dados de ausência (0) e presença (1) nas localidades são incontestes, dando confiabilidade e consistência aos modelos de regressão logística. As elevadas concordâncias entre as probabilidades previstas e observadas nos modelos de regressão logística asseguram a confiabilidade dos modelos e das análises feitas no presente estudo.

As distribuições geográficas não são determinadas por um único fator (biótico ou abiótico), sendo ainda influenciadas por processos de diferentes escalas (históricas e ecológicas) (ENDLER 1982, Myers \& Giller 1988a, b, Cerqueira 1995). Neste estudo foi investigada a importância do clima e das eco-regiões como determinantes da distribuição de C. flaviceps.

As análises mostraram que os fatores climáticos parecem limitar a distribuição de $C$. flaviceps. O resultado das análises dos fatores climáticos na distribuição de $C$. flaviceps, sintetizadas nas figuras 3 e 4, sugeriram que existiriam duas áreas com maiores probabilidades (> 40\%) de ocorrência para esta espécie. Os pontos de coleta de $C$. flaviceps estão exatamente localizados dentro de uma destas áreas de alta probabilidade (Fig. 4). A outra área com maior probabilidade, situada à NW da primeira, faz parte exatamente da distribuição de C. geoffroyi (Fig. 5) não sendo, portanto, passível de ocorrer C. flaviceps. As espécies de Callithrix endêmicas da Mata Atlântica são parapátricas, não havendo registro de simpatria das formas parentais (VIVo 1991). Aparentemente, a presença de uma espécie de Callithrix impede que outra espécie ocorra na mesma localidade, embora as espécies invasoras (C. Jacchus e C. Penicillata) possam ocorrer em simpatria com algumas das outras espécies de Callithrix. Contudo, esta questão deve ser vista com cautela, desde que não existem estudos sobre qual a importância das interações interespecíficas nos padrões de distribuição parapátrica dos Callithrix.

As figuras 1 e 4 sugerem que existe uma sobreposição nas distribuições climáticas preditas para C. flaviceps e as outras três espécies. A idéia corrente é a de que as flutuações climáticas ocorridas do final do Terciário e ao longo do Quaternário, quando as matas se fragmentaram e coalesceram, tiveram importância no processo de especiação de algumas espécies de calitriquídeos (CERqueira 1982, Kinsey 1982). Embora existam dúvidas sobre se ocorreram ou não especiações durante o Quaternário, ninguém discorda com a existência das flutuações climáticas ocorridas durante este período, não havendo controvérsias sobre o papel destas flutuações nos padrões de distribuição das espécies (Cerqueira 1982, Brown 1987, Colinvaux 1996, Rylands et al. 1996). Seguindo a linha de raciocínio de que as flutuações climáticas ocorridas durante o Quaternário interromperam o fluxo gênico entre as espécies de Callithrix, é possível que a zona híbrida entre algumas espécies, como $C$. flaviceps e $C$. aurita, seja o resultado de um contato secundário entre elas, proporcionado pela sobreposição da distribuição climática destas duas espécies. 

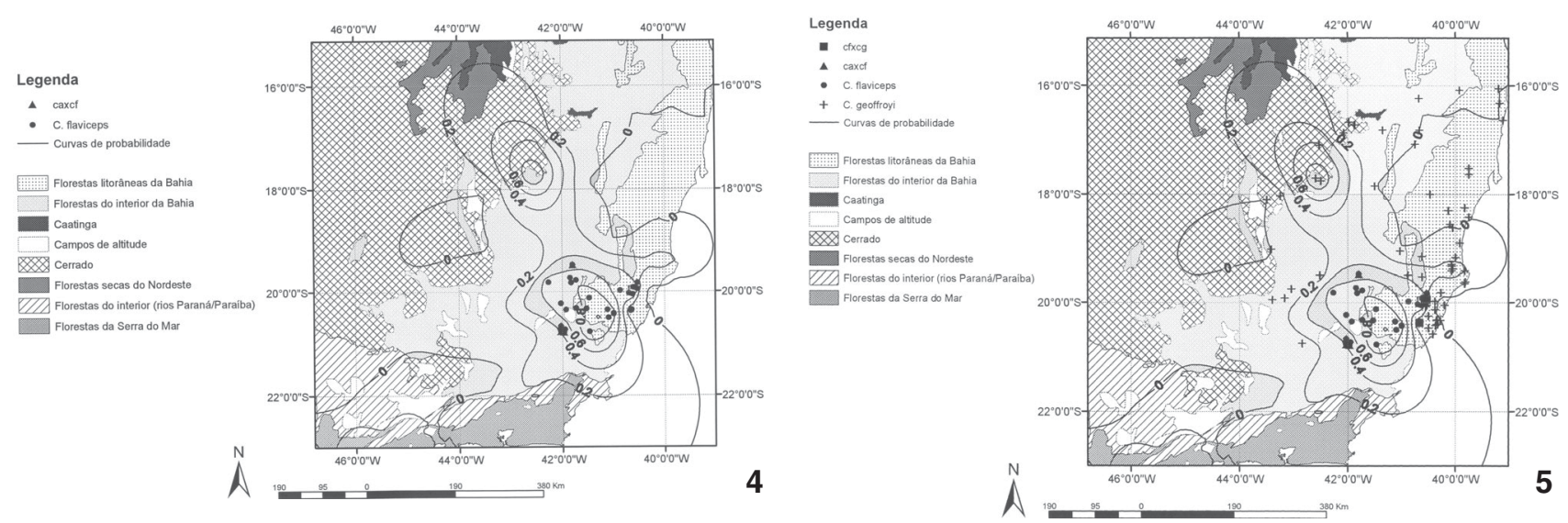

Figuras 4-5. Localidades de ocorrência de: (4) C. flaviceps - parentais e híbridos entre C. aurita e C. flaviceps (caxcf) - e (5) - parentais e dos híbridos entre C. flaviceps e C. geoffroyi e (cfxcg) e entre C. aurita e C. flaviceps (caxcf) -, e a distribuição predita (probabilidades de 0 a 1) para C. flaviceps a partir das variáveis climáticas selecionadas no modelo de regressão logística (veja o texto) e as eco-regiões.

Estudos anteriores argumentaram que os fatores climáticos e a vegetação seriam tão importantes quantos rios como barreiras geográficas para as espécies de Callithrix do grupo jacchus (Hershrovitz 1977, Cerqueira 1982, Kinsey 1982, Rylands et al. 1996). As análises apresentadas no presente estudo demonstraram que a distribuição de $C$. flaviceps pode ser de fato influenciada por diferentes fatores. Dificilmente um único fator, e portanto uma única análise, poderá explicar a distribuição de qualquer espécie. A investigação de vários fatores ao mesmo tempo, estudando e comparando espécie por espécie, parece ser a estratégia adequada para se entender os fatores determinantes das distribuições geográficas das espécies.

Concluindo, análises feitas a partir de fatores ambientais, como clima e vegetação, possibilitam que se façam mapas com hipóteses sobre os limites das distribuições geográficas das espécies. Tais análises podem ainda gerar informações importantes para a conservação e monitoramento das populações de diversas espécies.

\section{AGRADECIMENTOS}

Mario de Vivo, Lena Geise, Marcus V. Vieira, Ronaldo Fernandes, Cibele Bonvicino, Kátia Torres, Simone Freitas e Paulo Cordeiro fizeram uma série de comentários e críticas a uma versão preliminar deste trabalho. CNPq, FAPERJ e PROBIO(MMA-GEF) financiaram este estudo.

\section{REFERÊNCIAS BIBLIOGRÁFICAS}

Andrew, P. \& E.M. O’Brien. 2000. Climate, vegetation, and predictable gradients in mammal species richness in southern Africa. Journal of Zoology, London, 251: 205-231.

Bergallo, H.G.; L. Geise; C.R. Bonvicino; R. Cerqueira; P.S. D'Andrea; C.E. Esberard; F.A.S. Fernandez; C.E.V. Grelle; S. Siciliano \& S.M. VAz. 2000. Mamíferos, p. 125-135. In: H.G. Bergallo; C.F.D.
Rocha; M. van Sluys; L. Geise \& M.A. Alves (Eds). Lista da Fauna Ameaçada do Estado do Rio de Janeiro. Rio de Janeiro, EdUERJ, 205p.

BRASIL. 1969. Atlas Climatológico do Brasil. Rio de Janeiro, Ministério da Agricultura, ECEPLAN, Escritório de Meteorologia, 100p.

Brown, K.S. 1987. Conclusions, synthesis, and alternative hypothesis, p. 175-196. In: T.C. Whitmore \& G.T. Prance. (Eds). Biogeography and quaternary history in Tropical America. Oxford, Clarendon Press, 214p.

Bustamante, J. 1997. Predictive models for Lesser Ketrel (Falco naumanni): distribution, abundance and extinctions. Biological Conservation, Devon, 80: 153-160.

Cerqueira, R. 1982. South American Landscapes and their Mammals, p. 53-76. In: M.A. Mares \& H.H. Genoways (Eds). Mammalian Biology in South America. Linesville, Pymatuning Laboratory of Ecology, University of Pittsburgh, Special Publications Series, 539p.

Cerqueira, R. 1985. The distribution of Didelphis (Poliprotodontia, Didelphidae) in South America. Journal of Biogeography, London, 12: 135-145.

Cerqueira, R. 1995. Determinação de distribuições potenciais de espécies, p. 141-161. In: P. Peres-Neto; J.L. Valentin \& F.A.S. Fernandes. (Eds). Oecologia Brasiliensis. Rio de Janeiro, Programa de Pós-Graduação em Ecologia, Universidade Federal do Rio de Janeiro, vol. 2, 161p.

Cerqueira, R.; G. Marroig \& L. Pinder. 1998. Marmosets and Liontamarins distribution (Callitrichidae, Primates) in Rio de Janeiro, South-eastern Brazil. Mammalia, Paris, 62: 213-226.

Colinvaux, P. 1996. Quaternary environmental history and forest diversity, p. 359-406. In: J.B. JACKSON; A.F. BUDD \& A.G. COATES (Eds). Evolution and environmental in Tropical America. Chicago, The University of Chicago Press, 425p.

Correa, K.M.; P.E.G. Coutinho \& S.F. Ferrari. 2000. Between-year 
differences in the feeding ecology of highland marmosets (Callithrix aurita and Callithrix flaviceps) in south-eastern Brazil. Journal of Zoology, London, 252: 421-427.

Cueto, V.R. \& J.L. Casenave. 1999. Determinants of bird species richness: role of climate and vegetation structure at a regional scale. Journal of Biogeography, London, 26: 487-492.

Dinerstein, E; D.M. Olson; D.J. Graham; A.L. Webster; S.A. Primm; M.P. Bookbinder \& G. Ledec. 1995. A Conservation Assessment of the terrestrial ecoregions of Latin America and the Caribbean. Washington, WWF, World Bank, 129p.

ENDLER, J.A. 1977. Geographic variation, speciation, and clines. Princeton, Princeton University Press, 246p.

ENDLer, J.A. 1982. Problems in distinguishing historical from ecological factors in biogeography. American Zoologist, Seatle, 22: 441-452.

ESRI. 2002. ArcMap 8.2. Redlands, Environmental Systems Research Institute.

Ferrari, S.F.; K.M. Correa \& P.E.G. Coutinho. 1996. Ecology of "Southern" marmosets (Callithrix aurita and Callithrix flaviceps): how different, how similar?, p. 157-171. In: M. NorConK; A. Rosenberger \& P. Garber. (Eds). Adaptive radiations of Neotropical Primates. New York, Plenum Press, 348p.

Fonseca, G.A.B.; A.B. Rylands; C.M.R. Costa; R.B.Machado \& Y.L.R. LEITE. 1994. Livro vermelho dos mamíferos brasileiros ameaçados de extinção. Belo Horizonte, Fundação Biodiversitas, 459p.

Golden. 2002. Surfer, Version 8.0. Colorado, Golden Software. Grelle, C.E.V. 2003. Forest structure and vertical stratification of small mammal populations in a secondary forest, southeastern Brazil. Studies on Neotropical Fauna \& Environment, Lisse, 38: 81-85.

Hershrovitz, P. 1977. Living New World monkeys (Platyrrhini). Chicago, University of Chicago Press, vol. 1, 1117p.

Hill, M.O. 1991. Patterns of species distribution in Britain elucidated by canonical correspondence analysis. Journal of Biogeography, London, 18: 247-255.

Hosmer, D.W. \& S. Lemeshow. 1989. Applied Logistic Regression. New York, John Wiley, 307p.

IBge. 1993. Mapa de vegetação do Brasil. Rio de Janeiro, Ministério do Planejamento, Instituto Brasileiro de Geografia e Estatística IBGE.

JaCkson, S.M. \& A. Claridge. 1999. Climatic modelling of the distribution of the mahogany glider (Petaurus gracilis), and the squirrel glider ( $P$. norfolcensis). Australian Journal of Zoology, Collingwood, 24: 47-57.

Jefrree, E.P. \& C.E. Jefreee. 1994. Temperature and the biogeographical distribution of species. Functional Ecology, Oxford, 8: 640-650.

KINSEY, W.G. 1982. Distribution of primates and forest refuges, p. 455-482. In: G.T. PRANCE (Ed.). Biological diversification in the tropics. New York, Columbia University Press, 714p. LAW, B.S. 1994. Climatic limitation of the southern distribution of the common blossom bat Syconycteris australis in New South Wales. Australian Journal of Zoology, Collingwood, 19: 366-374.

Lindnmayer, D.B.; H.A. Nix; J.P. McMahon; M.F. Hutchinson \& M.T. T.ANTON. 1991. The conservation of leadbeater's possum, Gymnobelideus leadbeateri (McCoy): a case study of the use of bioclimatic modelling. Journal of Biogeography, London, 18: 371-383.

Machado, A.B.M.; G.A.B. Fonseca; R.B. Machado; L.M.S. Aguiar \& L.V. Lins. 1998. Livro vermelho das espécies ameaçadas de extinção da fauna de Minas Gerais. Belo Horizonte, Fundação Biodiversitas, 605p.

Manel, S.; J.M. Dias; S.T. Buckton \& S.J. Ormerod. 1999. Alternative methods for predicting species distribution: an ilustration with Himalayan river birds. Journal of Applied Ecology, Oxford, 36: 734-747.

Manly, B.F.J.; L.L. McDonald \& D.L. Thomas. 1993. Resource Selection by animals: statistical design and analysis for field studies. New York, Chapman \& Hall, 177p.

McKenney, W.; B.G. Mackey; J.P. Bogart; J.E. Mckee; M.J. Oldham \& A. С Hек. 1998. Bioclimatic and spatial analysis of Ontario reptiles and amphibians. Écoscience, Laval, 5: 18-30.

Myers, A.A. \& P.S. Giller. 1988a. Process, pattern and scale in biogeography, p: 3-12. In: A.A. Myers \& P.S. Giller (Eds). Analytical Biogeography. London, Chapman \& Hall, 578p.

Myers, A.A. \& P.S. Giller. 1988b. Biogeographic patterns: introduction, p: 15-21. In: A.A. Myers \& P.S. Giller (Eds). Analytical Biogeography. London, Chapman \& Hall, 578p.

NAToRi, M. 1990. Numerical analysis of systematics status of Callithrix kuhli based on measurements of the postcanine dentition. Primates, Aichi, 31: 555-562.

NATORI, M. 1994. Craniometrical variation among eastern Brazilian marmosets and their systematic relationships. Primates, Aichi, 35: 167-176.

Nix, H. 1986. A biogeographical analysis of the Australian elapid snakes, p. 4-15. In: R. LONGMORE. (Ed.). Atlas of elapid snakes. Canberra, Australian Government Publishing Service, Australian Flora and Fauna Series, no. 7, 115p.

NoRTh, M.P. \& J.H. REYNOLDS. 1996. Microhabitat analysis using radiotelemetry locations and polytomous logistic regression. Journal of Wildlife Management, Bethesda, 60: 639-653.

PAINTER, R.A. \& M.A. TAYLOR. 1991. Ornithological Gazeteer of Brazil. Massachusetts, Harvard University, 788p.

RAPOPORT, E.H. 1982. Areography: geographical strategies of species. Oxford, Pergamon Press, 269p.

Rodriguez, A. \& H. ANDrén. 1999. A comparison of Eurasian red squirrel distribution in different fragmented landscapes. Journal of Applied Ecology, Oxford, 36: 649-662.

Rylands, A.B.; G.A.B. Fonseca; Y.L. Leite \& R. Mittermeier. 1996. Primates of the Atlantic Forest: Origin, Distribution, Endemism, and Communities, p: 21-51. In: M. NorConK; A. RosenBERGER \& P. GARBER (Eds). Adaptive radiations of Neotropical primates. New York, Plenum Press, 348p.

Revista Brasileira de Zoologia 23 (2): 414-420, junho 2006 
Scott, J.M.; P.J. Heglund; M.L. Morrison; J.B. Haufler; M.G. Raphael; W.A. Wall \& F.B. Samson. 2002. Predicting species occurences: issues of accuracy and scale. Washington, Island Press, 868p.

TAYlor, R.A.J. \& L.R. TAYLOR. 1979. A behavioural model for the evolution of spatial dynamics, p: 1-27. In: R.M. ANDERSON; B.D. Turner \& L.R. Taylor (Eds). Population dynamics. London, Blackwell, 298p.

United States. 1963. Official standard names. Washington, United States Board on Geographical Names, Office of Geography, Department of Interior, 915p.
VANZOLInI, P.E. 1970. Zoologia sistemática, geografia e a origem das espécies. São Paulo, IGEO/USP, 56p.

VAnZolini, P.E. 1992. A supplement to the ornithological gazetteer of Brazil. São Paulo, Museu de Zoologia, Universidade de São Paulo, 252p.

VIVo, M. 1991. Taxonomia de Callithrix Erxleben, 1777 (Callitrichidae, Primates). Belo Horizonte, Fundação Biodiversitas, 105p.

Walker, P.A. 1990. Modelling wildlife distributions using a geographic information system: kangaroos in relation to climate. Journal of Biogeography, London, 17: 279-289.

Recebido em 12.VIII.2005; aceito em 02.V.2006. 